\title{
PROPUESTA PARA EL PROYECTO ENERGÉTICO EN LA INSTITUCIÓN ETNOEDUCATIVA RURAL INTERNADO DE NAZARETH
}

Jesús Antonio Valencia Plaza, Lida Soraida Lucano Mafla, Francisco Giovanni Ortega Palacios, Erika Sarria Navarro, Carlos Mario Giraldo Yepes, Andrés Mauricio González Moreno

Semillero Select

Grupo de Investigación Inteligo

Institución Universitaria Antonio José Camacho

Recepción: 24/01/2019. Aceptado: 26/03/2019.

Cómo citar este artículo:

Valencia Plaza J.A., Lucano Mafla L.S., Ortega Palacios F.G., Sarria Navarro E., Giraldo Yepes C.M. \& González Moreno A.M. (2019). Propuesta para el proyecto energético en la Institución Etnoeducativa Rural Internado de Nazareth. Revista Sapientía, 11 (21), 6 - 19.

\section{RESUMEN}

El presente artículo describe un análisis del estado actual del suministro energético de la Institución Etnoeducativa Rural Internado de Nazareth, ubicada en el corregimiento de Nazareth, en la alta Guajira. Por su característica de internado y la gran cantidad de personas que debe mantener diariamente, el actual servicio público de energía resulta insuficiente y limita el desarrollo de las actividades personales, académicas y administrativas de la comunidad.

Se evidencia, entonces, la posibilidad de implementar un proyecto basado en energías renovables, que ofrezca un suministro de energía durante todo el día, y no solo unas horas como se realiza actualmente, y que se constituya en una opción adecuada para una población con un alto nivel de pobreza y debilidad institucional, sumado a condiciones de sequía extrema. Por tanto, este documento pretende convertirse en un referente que motive el desarrollo de soluciones desde el punto de vista energético para la zona de la Alta Guajira

El diseño planteado finalmente involucra soluciones híbridas que combinan energía solar fotovoltaica, con una planta eléctrica diésel presente en el Internado, permitiendo un aprovechamiento de los recursos existentes, o una solución que reemplaza la planta eléctrica por un aerogenerador para el uso exclusivo de energías limpias.

\section{PALABRAS CLAVES}

Fuentes de energía renovables, análisis energético, sistemas fotovoltaicos.

\section{ABSTRACT}

This article describes an analysis of the current state of the energy supply of the Institución Etnoeducativa Rural Internado de Nazareth, in the High Guajira. For being a boarding school and the large number of people it must maintain daily, the current public energy service is insufficient and limits the development of the personal, academic and administrative activities of the community. 
It's evident the possibility of implementing a solution based on renewable energies, which offers a supply of energy throughout the day, and not just a few hours as is currently done, and which constitutes an adequate solution for a population with a high level of poverty and institutional weakness, added to conditions of extreme drought. Therefore, this document aims to become a reference that motivates the development of solutions from the energy point of view for the area of Alta Guajira

The proposed design finally involves hybrid solutions that combine photovoltaic solar energy, with a diesel power plant present in the boarding school, allowing an exploitation of existing resources, or a solution that replaces the power plant with a wind turbine for the exclusive use of clean energies.

\section{KEYWORDS}

Renewable energy sources, energy analysis, photovoltaic systems.

\section{INTRODUCCIÓN}

Debido al aislamiento de la zona de la alta Guajira Colombiana, y a las condiciones ambientales y sociales que se presentan en estos territorios, existen dificultades en cuanto al servicio de energía. Específicamente el corregimiento de Nazareth presenta una red de distribución de energía que solo funciona unas pocas horas del día, necesitándose otras alternativas energéticas, que en la mayoría de los casos son plantas eléctricas de diésel, para poder cumplir con los requerimientos del diario vivir, con el claro sobrecosto que implica depender de un sistema alimentado por combustible fósil.

Ubicado en esta región y con las múltiples dificultades asociadas a su objetivo social, se encuentra la Institución Etnoeducativa Rural Internado de Nazareth. Esta lleva más de 100 años de servicio, fue fundada el 6 de agosto del año 1911, y cumple con una misión pedagógica y social en una comu- nidad perteneciente a la etnia Wayuu, la etnia indígena con mayor cantidad de población del país según el DANE (Ministerio de Cultura. República de Colombia, 2010). Actualmente la institución es operada por las hermanas de la Comunidad Hermanas Terciarias Capuchinas (Atlas Amigoniano, 2009) (Jiménez Pérez, 2015) (Arbeláez Jiménez \& Vélez Posada, 2008).

El objetivo de esta investigación fue la generación de una propuesta de solución, enfocada desde la generación de energía renovable, que permita suplir los requerimientos energéticos de la Institución Etnoeducativa Rural Internado de Nazareth. Al respecto es pertinente tener en cuenta que la solución propuesta debe permitir un margen de crecimiento de usuarios y, por tanto, de gasto de energía.

\section{MARCO LEGAL}

Desde la academia, las empresas y el gobierno nacional se han adelantado diferentes estudios tendientes a describir y entender el efecto que tendría en la matriz energética de Colombia el acceso a las Fuentes No Convencionales de Energía Renovable (FNCER). En Colombia son considerados FNCER las siguientes fuentes: biomasa, aprovechamientos hidroeléctricos de baja cobertura, energía eólica, fotovoltaica, geotérmica y mareomotriz (Fernández Robles, 2017).

Este interés tiene como punto de partida la búsqueda de una alternativa a la gran dependencia que tiene el país de los combustibles fósiles y de los recursos hidroenergéticos. Según datos del Ministerio de Minas y Energía correspondientes al año 2015, el 78\% del consumo energético está basado en recursos de origen fósil con una disponibilidad cada vez menor (una oferta de 7 a 15 años). En cuanto a la generación eléctrica anual, entre el 70\% y el $80 \%$ se obtiene de hidroeléctricas cuya producción se ven afectada por fenómenos como El Niño y el cambio climático (Unidad de Planeación Minero Energética - UPME, 2015). 
A partir de este panorama se genera el Plan de Expansión de Referencia Generación Transmisión 2015-2029, documento estratégico para la planificación de los recursos de generación y la expansión de las redes de transmisión eléctrica a nivel nacional, que define 8 escenarios que integran diferentes fuentes de generación eléctrica, donde por ejemplo se destacan entre las FNCER la producción eólica, ubicada principalmente en La Guajira (Unidad de Planeación Minero Energética (UPME), 2017).

\section{MARCO GEOGRÁFICO}

Un aspecto importante para considerar es la difícil geografía de la zona donde se encuentra ubicado el Internado. Nazareth es un corregimiento de la Alta Guajira separado de Uribia, su cabecera municipal, por un trayecto terrestre que toma aproximadamente 8 horas, donde no existen vías de acceso pavimentadas $y$, por ende, se accede únicamente a través de camperos adecuados para el paso por el desierto (Ramírez Álvarez, 2015). En la Fig. 1 puede observarse la ubicación del corregimiento de Nazareth.

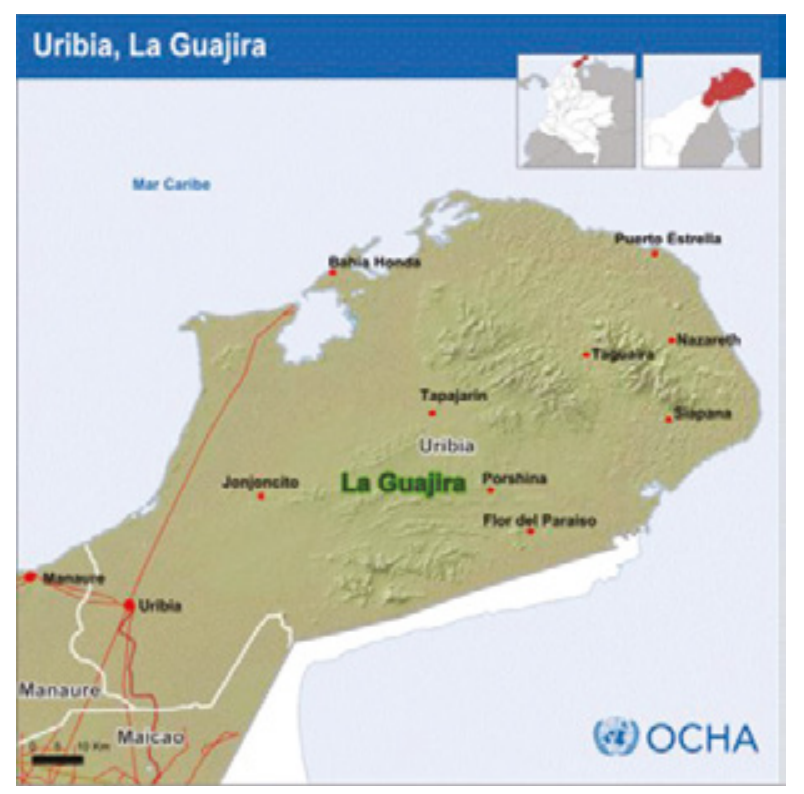

Fig. 1. Mapa del Municipio de Uribia y del Corregimiento de Nazareth. (Burbano C. \& Ocha M. B., 2014)
La densidad de energía eólica en la península de La Guajira a 20 metros de altura está entre entre 1.000 y $1.331 \mathrm{~W} / \mathrm{m} 2$, mientras que a 50 metros de altura está entre 2.197 y $2.744 \mathrm{~W} / \mathrm{m} 2$ (UPME - IDEAM, 2006). En cuanto a la generación de energía fotovoltaica, en la región noreste de la costa atlántica colombiana (La Guajira) se cuenta con el mayor potencial solar promedio diario del país con hasta $6 \mathrm{kWh} / \mathrm{m} 2$ y una disponibilidad promedio de entre 1.980 a $2.340 \mathrm{kWh} / \mathrm{m} 2 /$ año (UPME - IDEAM, 2005).

\section{MARCO REFERENCIAL}

Las Zonas No Interconectadas (ZNI) son "los municipios, corregimientos, localidades y caseríos no conectados al Sistema Interconectado Nacional", donde "la prestación del servicio de energía se hace principalmente mediante plantas de generación diesel, paneles solares y pequeñas centrales hidroeléctricas" (CREG, s.f.). Con respecto al problema energético de las ZNI se han realizado diversos estudios que evalúan los consumos y necesidades energéticas de las comunidades y plantean diversas soluciones.

Ojeda, Candelo, \& Silva (2017) presentan un estudio sobre la percepción de la energía sostenible que tienen las comunidades indígenas de La Guajira, determinando las necesidades y aspiraciones energéticas de ellas, contrastándolas con las carencias actuales. De esta manera se evidencia la necesidad del desarrollo de proyectos en la región.

Por su parte, Figueroa \& Mejía (2014) realizan un proyecto basado en el uso de sistemas híbridos eólicos y solares en las ZNI y evidencian las mejoras en la calidad de servicio a la comunidad que un proyecto desarrollado en un centro etnoeducativo en La Guajira puede generar. 


\section{METODOLOGÍA}

Debido a la dificultad de acceso a la zona se contactó telefónicamente a la comunidad del Internado para realizar una encuesta que permitiera conocer la infraestructura actualmente instalada y estimar los consumos de energía que se generan en su funcionamiento diario.

Con estos datos se calculó la potencia consumida en $\mathrm{kWh}$ /día y, luego de realizar una revisión de los equipos comerciales disponibles y la infraestructura física de la institución, se planteó una solución mixta que apoya la actual infraestructura instalada con la generación de energía renovable.

\section{DESCRIPCIÓN GENERAL DEL INTERNADO}

En la Figura 2 puede observarse una vista frontal del Internado.

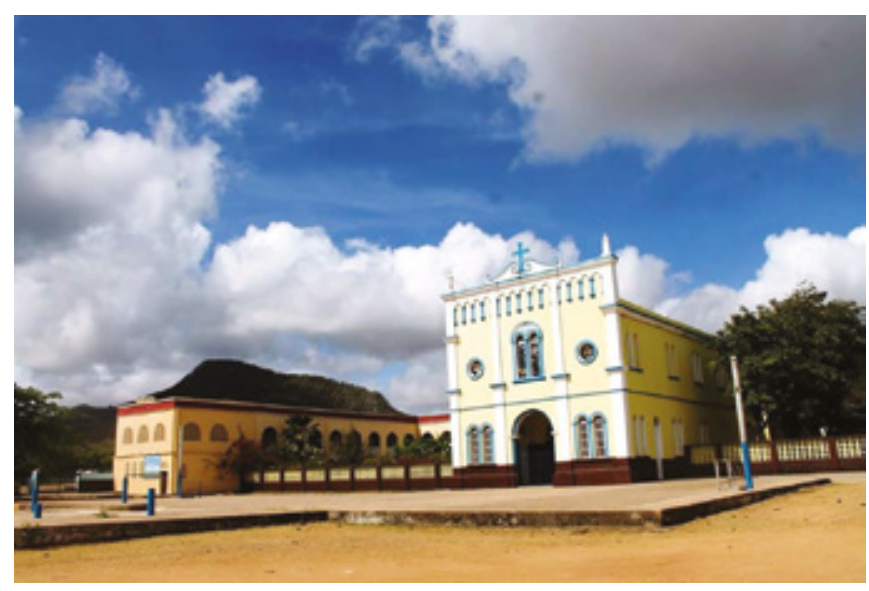

Fig. 2. Vista Frontal del Internado (Ecomochilas.com, 2014)

\section{HORARIO DE FUNCIONAMIENTO}

Las labores del internado comienzan a las 3:30 a.m., hora en la que se despierta la comunidad que permanece en el Internado, las labores habituales se desarrollan a lo largo del día y parte de la noche; las 8:00 p.m. es la hora en que los estudiantes van a dormir a sus habitaciones y 10:00 p.m. el momen- to en el que finalizan definitivamente las labores diarias.

La jornada académica va de 6:30 a.m. a 2:00 p.m., sin embargo, debe tenerse en cuenta que los estudiantes internos permanecen todo el día en la institución y, por tanto, en la tarde se realizan diversas actividades vocacionales, deportivas y lúdicas que involucran una gran parte de la comunidad estudiantil. Las salas de cómputo trabajan 22 horas a la semana cada una de ellas.

Asimismo es importante mencionar que las instalaciones del internado, y específicamente el auditorio, el polideportivo y los equipos de video y audio sirven a la comunidad de Nazareth en la realización de reuniones, eventos, celebraciones, cursos y demás actividades que contribuyen al desarrollo de la población.

\section{POBLACIÓN DE LA INSTITUCIÓN}

Durante el día el internado alberga 1000 personas entre docentes, religiosos y estudiantes, mientras que en horas de la noche y fines de semana permanecen 300 personas, de las cuales 260 son estudiantes.

\section{INFRAESTRUCTURA FÍSICA}

El internado cuenta con locaciones tanto para la realización de las actividades académicas diurnas como salones, auditorios, comedores, baños, y también dormitorios para la noche. En la Tabla 1 se listan los espacios indicando la utilización designada. 
Tabla 1.Locaciones del internado

\begin{tabular}{|c|c|}
\hline Descripción & Cantidad y Tipos \\
\hline \multirow{4}{*}{ Dormitorios } & $\begin{array}{l}2 \text { grupales, con capacidad para } 251 \text { estu- } \\
\text { diantes entre los dos }\end{array}$ \\
\hline & 8 para los docentes \\
\hline & 22 para trabajadores de diferentes áreas \\
\hline & 16 para la comunidad religiosa \\
\hline \multirow{3}{*}{ Aulas } & $\begin{array}{l}\text { Salón para trabajos artesanales (equivalen- } \\
\text { te a tres salones) }\end{array}$ \\
\hline & 25 salones de clase \\
\hline & 3 salas de cómputo \\
\hline \multirow{10}{*}{ Otras áreas } & 6 oficinas \\
\hline & 3 garajes \\
\hline & Carpintería \\
\hline & Cocina \\
\hline & Auditorio para presentaciones y reuniones \\
\hline & $\begin{array}{l}\text { Polideportivo con cancha de microfútbol } \\
\text { (cancha múltiple), }\end{array}$ \\
\hline & $\begin{array}{l}2 \text { comedores grandes, } 1 \text { mediano y } 1 \\
\text { pequeño }\end{array}$ \\
\hline & $\begin{array}{l}\text { Se proyecta un galpón de gallinas y pollos } \\
\text { para el próximo año }\end{array}$ \\
\hline & Área de caprinos (chivos, ovejos y cerdos), \\
\hline & Pasillos \\
\hline
\end{tabular}

\section{ESTADO ACTUAL DEL CONSUMO Y SUMINISTRO ENERGÉTICO}

\section{Estado de la Red Energética Pública}

Los corregimientos de Nazareth y Puerto Estrella reciben energía proveniente de una central diseñada con poli generación a partir de componentes de generación eólica, fotovoltaica y térmica-diesel, aunque en este momento, la generación eólica está fuera de servicio. Dicho sistema alimenta dos circuitos, uno con destino a Nazareth y otro a la población de Puerto Estrella. A pesar de que el objetivo de la construcción de esta central fue cubrir las 24 horas del día, los informes de telemetrías realizadas a 2016 detectan que el tiempo efectivo de prestación del servicio oscila entre 5 y 17 horas (Centro Nacional de Monitoreo-CNM, 2016), y el informe de fin de año del 2017 habla de un promedio de 9 horas de servicio.
En la Figura 3 pueden observarse datos relativos la prestación de servicio de energía eléctrica en el corregimiento de Nazareth consultados en el mapa web del Centro Nacional de Monitoreo de IPSE (Centro Nacional de Monitoreo, s.f.).

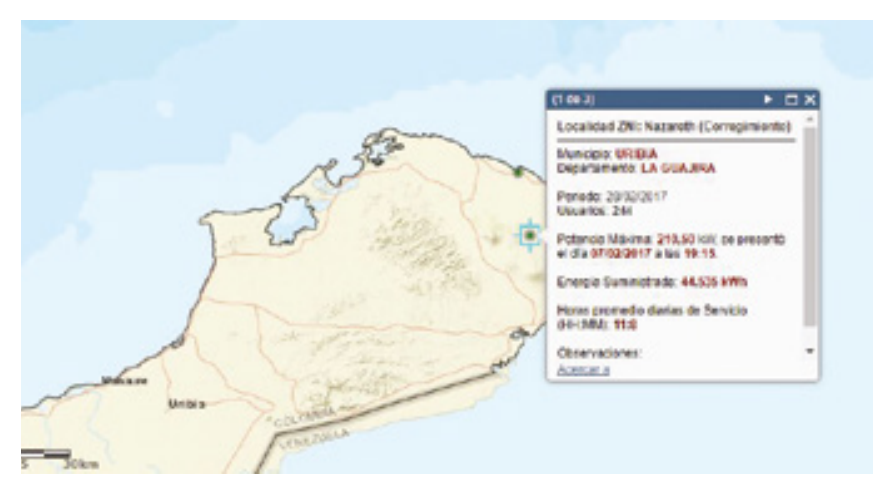

Fig. 3. Prestación del Servicio de Energía en el corregimiento de Nazareth. (SIGIPSE, 2018)

En la investigación realizada directamente con la comunidad del Internado se encontró que Nazareth recibe energía de la red pública de 9 a.m. a 2 p.m., sin embargo no hay garantía de prestación del servicio diariamente. Por esta razón, en este momento se utiliza una Planta Diesel de 100kVA, propiedad de la institución, para el resto de sus necesidades.

Esto significa que de las aproximadamente 19 horas diarias que hay actividad en el internado, solo 5 horas son cubiertas por la red de energía pública $y$, por tanto, la planta eléctrica diesel del internado debe cubrir las 14 horas de diferencia. La problemática radica en el costo del combustible necesario para operarla, que ocasiona que se limite su uso al mínimo; además, si se agota el combustible pensarse en el transporte de este desde los sitios de comercialización.

Sumado a lo anterior se presentan con mucha frecuencia cambios bruscos de voltaje y suspensiones que deterioran los equipos eléctricos y perjudican el funcionamiento normal de la instalación educativa, ya que se afecta la iluminación, ventilación, refrigeración de alimentos, reparaciones que involucren equipo eléctrico, y con ello, las actividades 
académicas y recreativas (Centro Nacional de Monitoreo - CNM, 2016).

\section{Carga Eléctrica Actual de la Institución}

Debido a los problemas en la recepción de la energía, las horas de uso de ventilación, iluminación y demás servicios no esenciales debe reducirse, dificultando los quehaceres habituales de la institución. Por ejemplo, el internado cuenta con ventiladores no instalados debido a que se debe priorizar el gasto de energía. En la Tabla 2 se presentan los consumos de energía eléctrica que se generarían por día, mes y año.

Tabla 2. Consumos Totales Calculados

\begin{tabular}{ll}
\hline \multicolumn{1}{c}{ Descripción } & Total \\
\hline Consumo promedio por día & $100,69 \mathrm{kWh} /$ día \\
\hline Consumo promedio por mes & $3,02 \mathrm{MWh} / \mathrm{mes}$ \\
\hline Consumo promedio por año & $36,75 \mathrm{MWh} / \mathrm{año}$ \\
\hline
\end{tabular}

En este momento la iluminación externa nocturna se limita en horas y, a nivel general, se utilizan bombillos fluorescentes e incandescentes con el gasto adicional que conllevan. En la Figura 4 se observa el perfil de consumo actual en kWh según la hora del día.

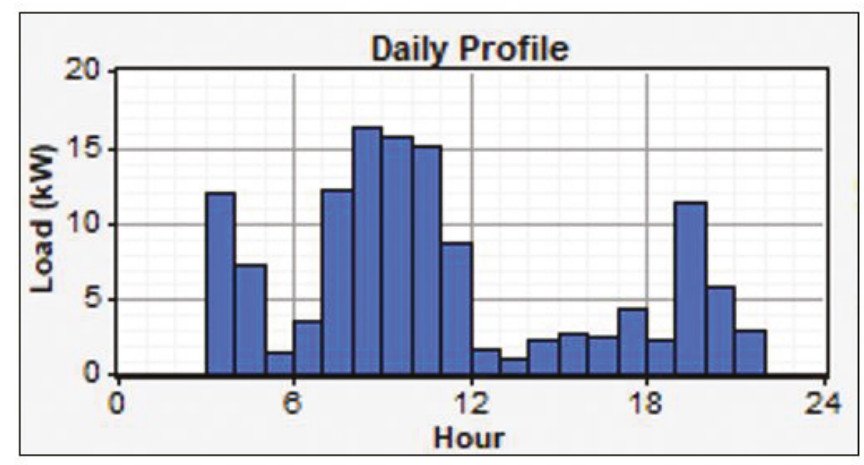

Fig. 4. Caracterización de la carga actual durante el día

Se observa que las horas de máximo consumo de energía corresponden al horario de clase regular, momento en el que se encuentra en el internado la totalidad de la población y en el que la mayoría de los espacios están en uso.

\section{DISEÑO DE LA SOLUCIÓN}

\section{Carga Eléctrica Proyectada}

Para la carga eléctrica proyectada se considera el uso óptimo de horas para una disponibilidad de energía las 24 horas, se eleva el número de ventiladores y, además, se considera el uso de iluminación de $12 \mathrm{~W}$, en lugar del que actualmente se maneja de $20 \mathrm{~W}$, contribuyendo a mejorar el gasto total de energía. En otros términos, se plantea tanto un aumento como un redireccionamiento de la energía a consumir.

Es importante mencionar que todos los valores correspondientes a la cantidad y tipo de elementos considerados en el cálculo de la carga eléctrica actual y proyectada por hora son establecidos con base en los horarios típicos de los residentes: los horarios de clase, alimentación, recreación, entre otras actividades, teniendo en cuenta el número de espacios de cada tipo. Por ejemplo, los salones de clase no se ocupan en la jornada nocturna a diferencia de los dormitorios, etc. En el Anexo A se presentan los cuadros de carga actual y los proyectados.

En la Tabla 3 se presentan los consumos de energía eléctrica que se generarían por día, mes y año para cubrir las necesidades completas del Internado, a fin de diseñar una solución mixta que incluya el uso de la infraestructura actual y un sistema de generación y almacenamiento de energías renovables.

Tabla 3. Consumos Totales Proyectados

\begin{tabular}{ll}
\hline \multicolumn{1}{c}{ Descripción } & Total \\
\hline Consumo promedio por día & $139,82 \mathrm{kWh} /$ día \\
\hline Consumo promedio por mes & $4,19 \mathrm{MWh} / \mathrm{mes}$ \\
\hline Consumo promedio por año & $51,03 \mathrm{MWh} / \mathrm{año}$ \\
\hline
\end{tabular}

En la Figura 5 se observa el aporte de cada tipo de carga al total de kWh y en la Figura 6 se observa el consumo de kWh según la hora del día. 


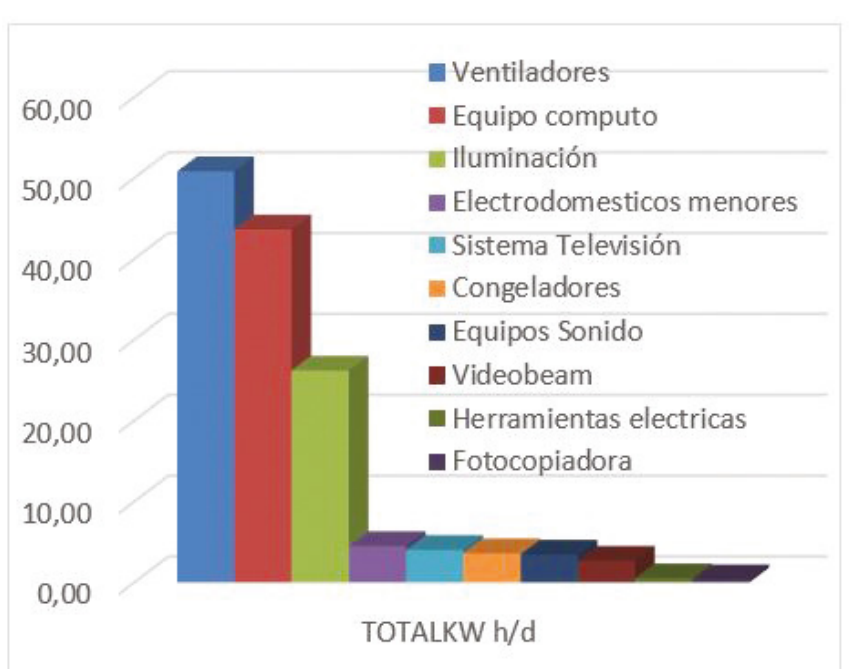

Fig. 5. Consumo de Energía por Categoría (kWh/día)

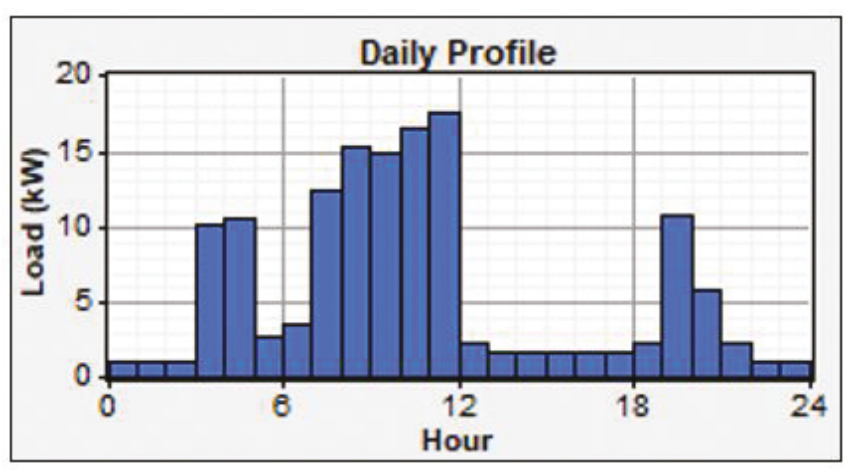

Fig. 6. Caracterización de la carga proyectada durante el día

\section{Potencial Eólico y Solar de Nazareth}

El poblado de Nazareth está ubicado en latitud $12^{\circ} 11^{\prime} 4,272^{\prime \prime} \mathrm{N}$ y longitud $71^{\circ} 17^{\prime} 20,459^{\prime \prime} \mathrm{W}$ con una altitud de $10 \mathrm{~m}$ sobre el nivel del mar. Estas coordenadas permiten obtener el potencial eólico y solar del municipio a partir de la base de datos atmosférica de la NASA (NASA, s.f.):

- La velocidad anual promedio del viento es de $6,21 \mathrm{~m} / \mathrm{s}$

- El potencial de radiación solar es de $6,49 \mathrm{kWh} /$ $\mathrm{m} 2 / \mathrm{d}$ en promedio durante el año, siendo diciembre el peor mes según se muestra en la Figura 7.

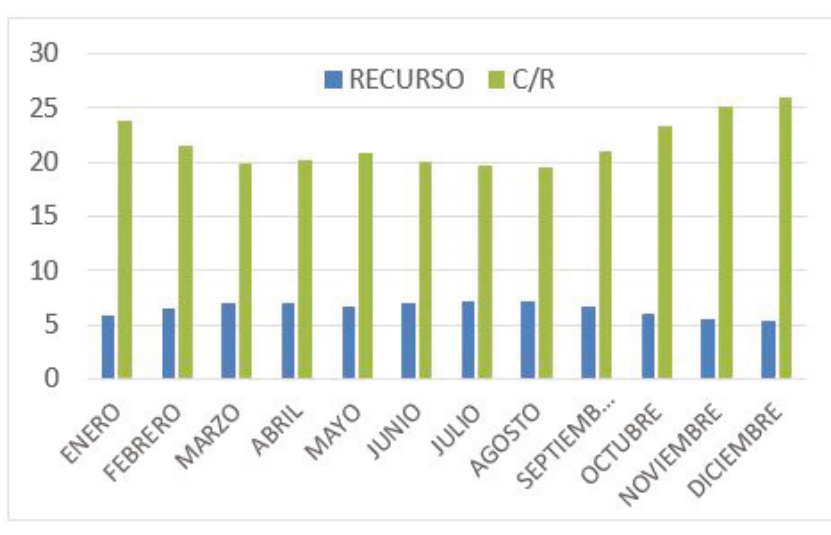

Fig. 7. Comparación recurso solar y consumo

\section{Consumo de Energía}

Como ya se ha dicho, la red pública solamente entrega energía 5 horas al día, la planta de diesel del internado cubre el resto del horario. El objetivo de la solución híbrida que se propone cubriría las horas sin servicio y reduciría a un $10 \%$ del total de energía necesaria el consumo de la red pública en las horas con suministro.

En la Figura 8 se realiza una comparación entre la energía suministrada por la red, el consumo actual y el consumo con la solución propuesta.

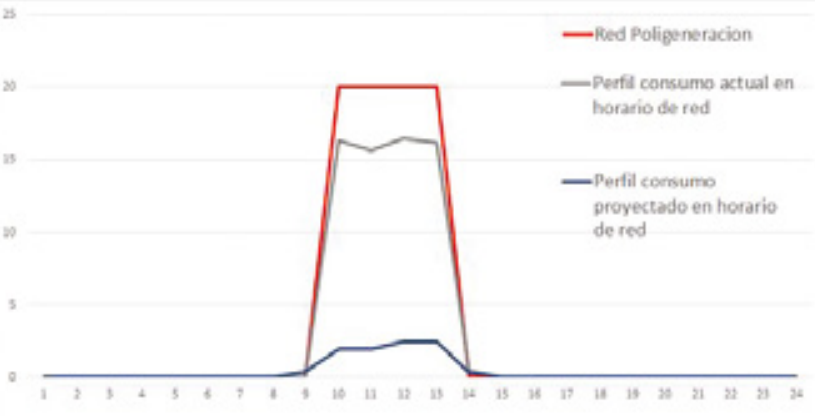

Fig. 8. Perfil de consumo de la red eléctrica pública actual y proyectada

Siguiendo con este análisis, la Figura 9 compara el perfil de consumo actual de la energía generada por la planta diésel del Internado y el perfil de consumo con la solución híbrida propuesta. 


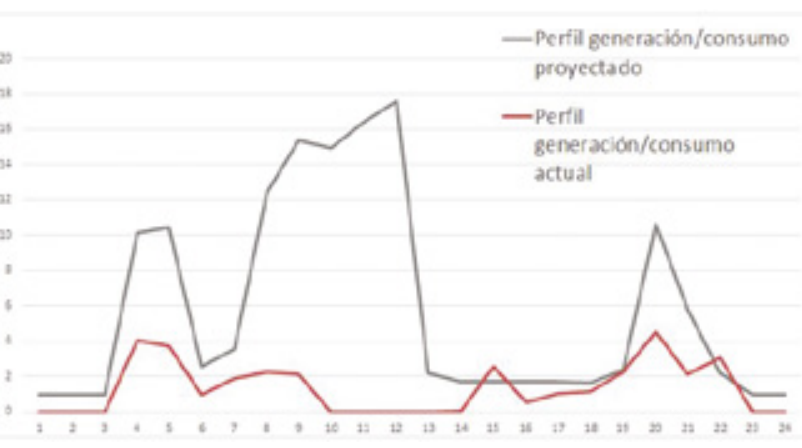

Fig. 9. Comparación entre el perfil de consumo de la planta diésel actual y la solución propuesta

\section{Decisiones de diseño}

Después de analizar las condiciones energéticas, de infraestructura, geográficas, etc., que configuran el caso del Internado Nazareth, se determinó que a pesar de que la red eléctrica pública llega a la zona, es inestable y los horarios en los que permanece activada pueden llegar a variar día a día. Por tanto, se plantea una solución híbrida tanto con energías renovables como con la conexión a la red pública, en la búsqueda de garantizar que en caso de faltar esta última, el sistema de energías renovables cubra todos los requerimientos de la Institución. En caso de presentarse el suministro de energía de la red pública, está se conecta al sistema gracias a las características del inversor híbrido, mejorando las condiciones de funcionamiento del sistema.

\section{Equipos Considerados para la Simulación}

A través de la herramienta Homer se realiza una simulación que involucra paneles solares, aerogeneradores, baterías y la planta de energía diésel ya existente en el Internado. De esta manera se evaluará si puede reusarse parte de la infraestructura ya existente. En la Figura 10 pueden observarse los equipos considerados para la simulación.

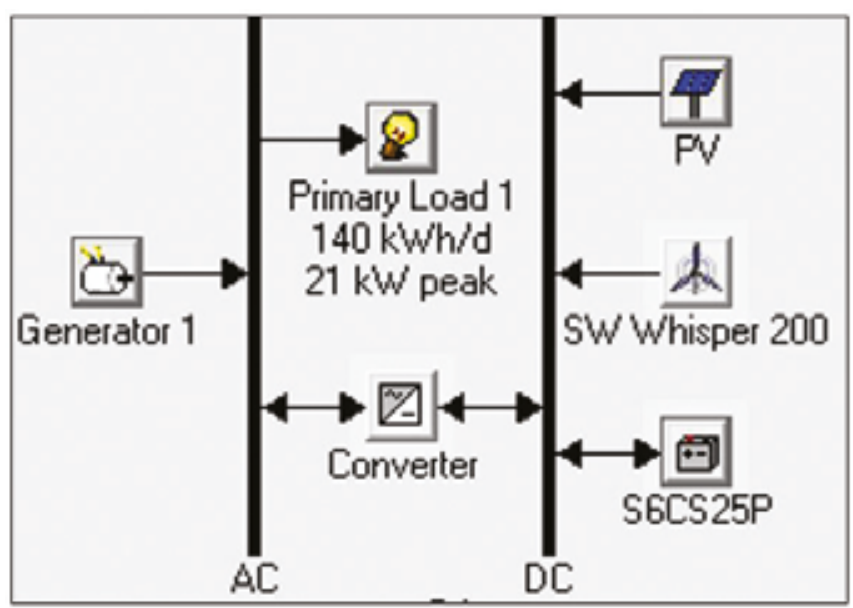

Fig. 10. Equipo por considerar en la simulación en Homer

Se utilizaron como variables de sensibilidad el aumento del precio del diésel y de la carga calculada en el Internado.

- Se consideraron aumentos en el precio del diésel del 21\% anual revisando el histórico de la región (SIPG, s.f.).

- Se consideraron aumentos de la carga del internado de un $10 \%$, lo cual permitiría simular, por ejemplo, la instalación de una nueva sala de computadores, por cada aumento.

Se debe tener en cuenta que, tal como ya se ha mencionado, no se incluirá la conexión a la red pública existente, debido a la inestabilidad del sistema actual. De este modo se garantiza un funcionamiento que no dependa de los cortes inesperados.

\section{Resultados de la Simulación}

Una vez realizada la simulación se obtiene la Figura 11 que relaciona las dos variables de sensibilidad consideradas: el precio del diésel y la carga. $\mathrm{Al}$ analizarla, se encuentra que para una carga de hasta $154 \mathrm{kWh} / \mathrm{d}$ la solución que solo integra paneles solares y baterías funcionaría, pero a partir de esa carga necesitaría el apoyo de la planta diésel. 


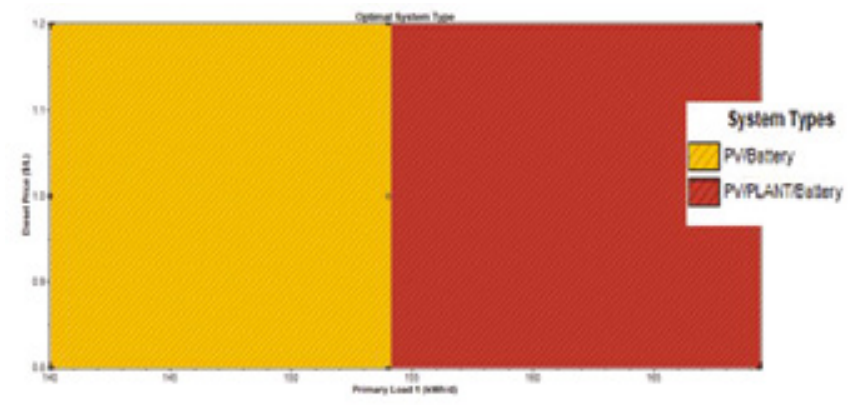

Fig. 11. Sistema Óptimo según las Variables de Sensibilidad

La lista completa de las configuraciones optimizadas generadas por Homer para la carga inicial de $140 \mathrm{kWh} / \mathrm{d}$ y el precio actual del diésel, de aproximadamente \$8400, se observa en la Figura 12.

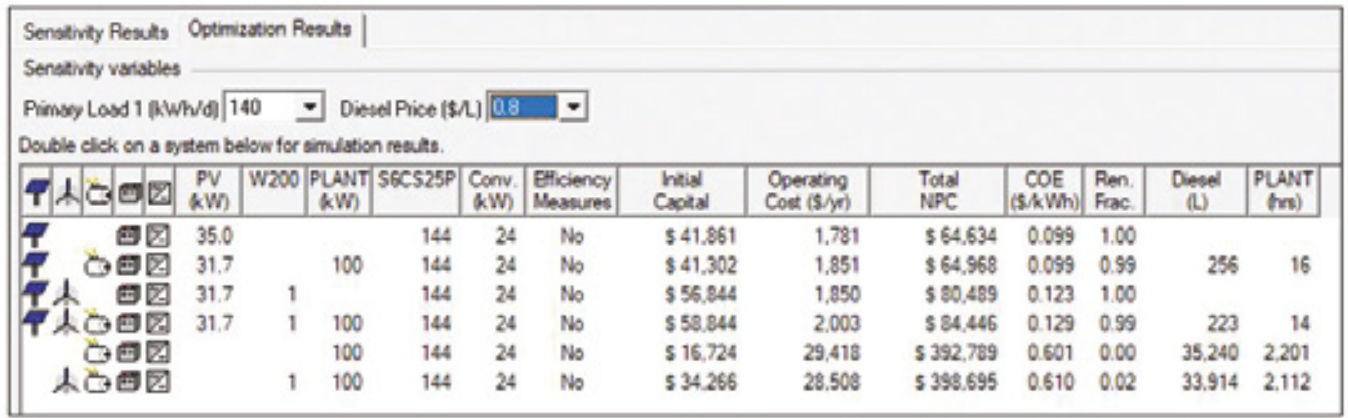

Fig. 12. Resultados optimizados Homer

A continuación, se describirán los pros y contras de los tres primeros resultados, a fin de seleccionar la mejor o las mejores opciones.

\section{Solución 1: Paneles Solares}

La primera solución considerada por Homer, incluye Paneles Solares y Baterías, pero es descartada al revisar los parámetros eléctricos que indican que se generaría un exceso de electricidad del 12.4\%, como se observa en la Figura 13:

\begin{tabular}{l|r|r|}
\multicolumn{1}{c|}{ Quantity } & $\mathrm{kWh} / \mathrm{yr}$ & \\
\hline Excess electricity & 8,386 & \\
\hline Unmet electric load & 0.0000961 & 0.0 \\
\hline Capacity shortage & 0.00 & 0.0 \\
\hline \multicolumn{1}{|c|}{ Quantity } & \multicolumn{2}{|c|}{ Value } \\
\hline Renewable fraction & \multicolumn{2}{|c|}{1.00} \\
\hline
\end{tabular}

Fig. 13. Exceso de Electricidad Generada para la Solución Paneles Solares 


\section{Solución 2: Paneles Solares y Planta Diésel} En esta solución se observa una infraestructura híbrida que permitiría aprovechar la planta diésel ya existente. Algunos de los aspectos más importantes de esta solución son:

- El exceso de electricidad es del 4.03\%, el cual está es un rango tolerable.

- La generación de energía es casi totalmente fotovoltaica (99\%), siendo la planta diésel un apoyo para la época del año con menos radiación solar, tal como se observa en la Figura 14.

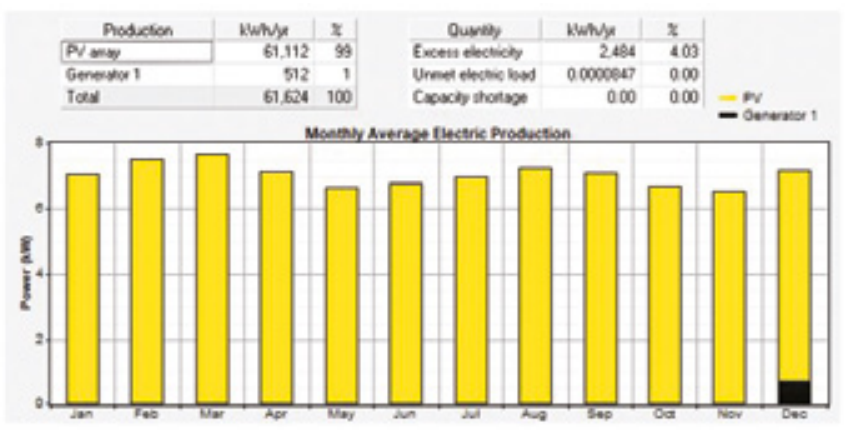

Fig. 14. Generación de energía para la Solución Solar-Diésel

- Al ser una solución que incluye combustibles fósiles, se presentan emisiones contaminantes como puede observarse en la Figura 15.

- El costo del $\mathrm{kWh}$ es de $\$ 280.67$, teniendo en cuenta que en una casa de estrato 3 en Cali el kWh tiene un costo de $\$ 483$, se obtiene un buen precio.

\begin{tabular}{|l|r|}
\multicolumn{1}{|c|}{ Pollutant } & Emissions $(\mathrm{kg} / \mathrm{yr})$ \\
\hline Carbon dioxide & 674 \\
\hline Carbon monoxide & 1.66 \\
\hline Unburned hydrocarbons & 0.184 \\
\hline Particulate matter & 0.125 \\
\hline Sulfur dioxide & 1.35 \\
\hline Nitrogen oxides & 14.8 \\
\hline
\end{tabular}

Fig. 15. Tabla Emisiones Contaminantes para la Solución Solar-Diésel
En cuanto a las baterías, puede observarse que es precisamente en los meses con menos potencial solar de la región (noviembre-diciembre), que alcanzan una descarga máxima del $40 \%$, como se observa en la Figura 16.

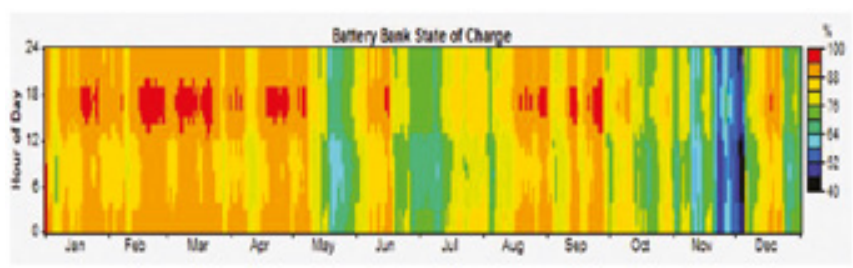

Fig. 16. Estado de la Carga del Banco de Baterías Solución Solar-Diésel

Solución 3: Paneles Solares y Aerogenerador En esta solución se observa una infraestructura híbrida que incluye paneles solares y la instalación de un aerogenerador, sin utilizar la planta diésel. Algunos de los aspectos más importantes de esta solución son:

- El exceso de electricidad es del 6.35\%, el cual está en un rango tolerable.

- La generación de energía es mayoritariamente fotovoltaica (97\%), siendo el aerogenerador un apoyo durante todo el año con un $3 \%$ de la producción de energía, tal como se observa en la Figura 17.

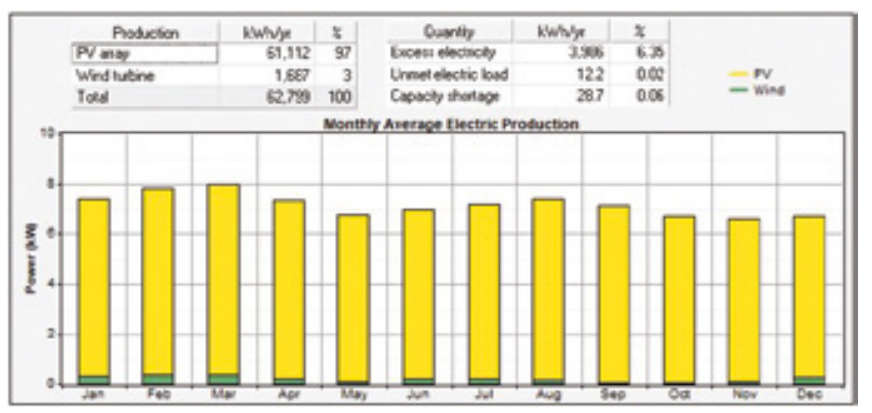

Fig. 17. Generación de energía para la Solución Solar-Viento

- El costo del $\mathrm{kWh}$ es de $\$ 348,71$ siendo aún un buen precio comparado con los costos de otras zonas del país. 
- Esta solución es completamente libre de contaminantes siendo, por tanto, el ideal de un futuro sin combustibles fósiles, y también para una zona cercana a un parque natural como lo es la Serranía de Macuira.

- En cuanto a las baterías, puede observarse que es precisamente en los meses con menos potencial solar de la región (noviembre-diciembre), que alcanzan una descarga máxima del $40 \%$, como se observa en la Figura 18.

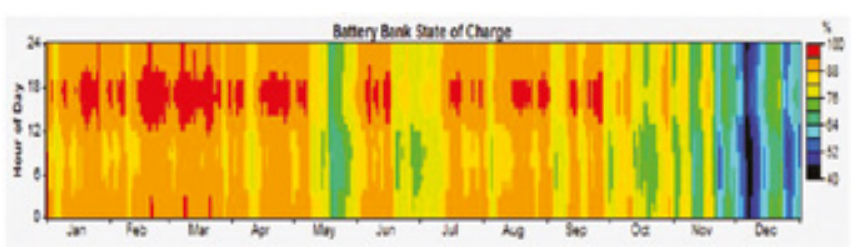

Fig. 18. Estado de la Carga del Banco de Baterías Solución Solar-Viento

\section{CONCLUSIONES}

La investigación realizada para el desarrollo de esta propuesta evidenció que, a pesar de esfuerzos realizados por los gobiernos regionales y nacionales, la zona de Nazareth no cuenta con un suministro estable de la red eléctrica pública. Gran parte de la documentación consultada asegura proveer hasta 11 horas diarias de suministro, pero la información entregada por la comunidad evidencia que no es así.

Con base en los resultados obtenidos en la simulación realizada, se encuentra que una solución híbrida debe ser la escogida. Las opciones incluyen la combinación Paneles solares/Planta Diésel o la combinación Paneles Solares/Aerogenerador. La selección entre estas opciones depende del deseo de aprovechar la infraestructura instalada u optar por una solución libre de emisiones contaminantes.

Adicionalmente en el caso de optar por la combinación Paneles solares/Planta Diesel, se ofrece un valor estimado del $\mathrm{kWh}$ de $\$ 280.67$, lo cual ofre- ce una rebaja del 19\% con respecto a una solución combinada de Paneles Solares/Aerogenerador.

El apoyo del banco de baterías, además de permitir el suministro de energía por fuera del horario de presencia de luz solar, cobra importancia en los meses de noviembre y diciembre, caracterizados por un menor potencial fotovoltaico y un menor potencial eólico.

\section{ANEXOS}

\section{Cuadros de Carga}

La Tabla 4 y la Tabla 5 presentan completamente detallados los cuadros de carga actual y deseado del Internado.

Tabla 4. Cuadro de Carga Actual

\begin{tabular}{|c|c|c|}
\hline Cantidad & Descripción & $\begin{array}{c}\text { Total } \\
\text { kWh/día }\end{array}$ \\
\hline 66 & Computadores Estudiantes & 28,29 \\
\hline 12 & $\begin{array}{l}\text { Computadores Administración y } \\
\text { Profesorado }\end{array}$ & 6,43 \\
\hline 80 & Tabletas & 0,80 \\
\hline 4 & Impresoras & 0,13 \\
\hline 1 & Fotocopiadora & 0,19 \\
\hline 3 & Proyectores & 1,07 \\
\hline 10 & Televisores Led & 1,56 \\
\hline 10 & Sistema satelital Direct TV & 1,12 \\
\hline 1 & Equipo sonido 1 & 2,21 \\
\hline 1 & Equipo sonido 2 (Gran Potencia) & 0,34 \\
\hline 3 & Congeladores & 5,13 \\
\hline 1 & Herramientas eléctricas & 0,51 \\
\hline 1 & Electrodomésticos menores & 2,70 \\
\hline 16 & Iluminación externa & 4,00 \\
\hline 176 & Iluminación dormitorios & 9,16 \\
\hline 6 & Iluminación cancha Múltiple & 0,51 \\
\hline 18 & Iluminación Oficinas & 1,03 \\
\hline 9 & Iluminación Garajes & 0,62 \\
\hline 3 & Iluminación Carpintería & 0,17 \\
\hline 6 & Iluminación Cocina & 0,72 \\
\hline 4 & Iluminación Galpones & 0,32 \\
\hline 60 & Iluminación Pasillos & 3,77 \\
\hline
\end{tabular}




\begin{tabular}{lll}
\hline 10 & Iluminación Iglesia & 0,80 \\
\hline 8 & Iluminación Comedor Grande & 0,32 \\
\hline 6 & Iluminación Comedor Mediano & 0,24 \\
\hline 4 & Iluminación Comedor Pequeño & 0,16 \\
\hline 24 & Iluminación Salas de computo & 2,06 \\
\hline 18 & Iluminación Auditorio multipropósito & 0,46 \\
\hline 50 & Iluminación Salones de clase & 4,29 \\
\hline 6 & Iluminación Salón trabajos artesana- & 0,17 \\
\hline 12 & les & 0,72 \\
\hline 181 & Iluminación baños comunes & 20,69 \\
\hline
\end{tabular}

Tabla 5. Cuadro de Carga Deseado

\begin{tabular}{|c|c|c|}
\hline Cantidad & Descripción & $\begin{array}{c}\text { Total } \\
\text { kWh/día }\end{array}$ \\
\hline 66 & Computadores Estudiantes & 35,357 \\
\hline 12 & $\begin{array}{l}\text { Computadores Administración y } \\
\text { Profesorado }\end{array}$ & 6,429 \\
\hline 80 & Tabletas & 1,600 \\
\hline 4 & Impresoras & 0,257 \\
\hline 1 & Fotocopiadora & 0,39 \\
\hline 3 & Proyectores & 2,68 \\
\hline 10 & Televisores Led & 2,34 \\
\hline 10 & Sistema satelital Direct TV & 1,68 \\
\hline 1 & Equipo sonido 1 & 2,76 \\
\hline 1 & Equipo sonido 2 (Gran Potencia) & 0,69 \\
\hline 3 & Congeladores & 3,60 \\
\hline 1 & Herramientas eléctricas & 0,51 \\
\hline 1 & Electrodomésticos menores & 4,50 \\
\hline 16 & Iluminación externa & 9,60 \\
\hline 176 & Iluminación dormitorios & 5,69 \\
\hline 6 & Iluminación cancha Múltiple & 0,51 \\
\hline 18 & Iluminación Oficinas & 1,11 \\
\hline 9 & Iluminación Garajes & 0,19 \\
\hline 3 & Iluminación Carpintería & 0,10 \\
\hline 6 & Iluminación Cocina & 0,58 \\
\hline 4 & Iluminación Galpones & 0,19 \\
\hline 60 & Iluminación Pasillos & 2,36 \\
\hline 10 & Iluminación Iglesia & 0,72 \\
\hline 8 & Iluminación Comedor Grande & 0,19 \\
\hline 6 & Iluminación Comedor Mediano & 0,14 \\
\hline
\end{tabular}

\begin{tabular}{lll}
\hline 4 & Iluminación Comedor Pequeño & 0,10 \\
\hline 24 & Iluminación Salas de computo & 1,23 \\
\hline 18 & Iluminación Auditorio multipropósito & 0,28 \\
\hline 50 & Iluminación Salones de clase & 2,57 \\
\hline 6 & $\begin{array}{l}\text { Iluminación Salón trabajos artesana- } \\
\text { les }\end{array}$ & 0,21 \\
\hline 12 & Iluminación baños comunes & 0,43 \\
\hline 181 & Ventiladores & 50,83 \\
\hline
\end{tabular}

\section{REFERENCIAS BIBLIOGRÁFICAS}

Arbeláez Jiménez, J., \& Vélez Posada, P. (2008). La Etnoeducación en Colombia. Una Mirada Indígena. Medellín: Universidad EAFIT. Obtenido de https://repository.eafit.edu.co/bitstream/handle/10784/433/ Juliana_ArbelaezJimenez_2008.pdf?sequence $=1$

Atlas Amigoniano. (2009). Internado Indígena de Nazareth. Obtenido de https://goo.gl/r4SdcB

Centro Nacional de Monitoreo - CNM. (2016). Informe Mensual de Telemetría 2016 Nazareth - Uribia - La Guajira. Recuperado el 25 de enero de 2018, de http://190.216.196.84/cnm

Centro Nacional de Monitoreo. (s.f.). Prestación de Servicio. Recuperado el 20 de Enero de 2018, de http://190.216.196.84/ps_cnm/

Congreso de la República de Colombia. (2014). Ley 1715 del 13 de mayo de 2014. Bogotá. Obtenido de http:// www.upme.gov.co/Normatividad/Nacional/2014/ LEY_1715_2014.pdf

CREG. (s.f.). Zonas no interconectadas. Obtenido de Comisión de Regulación de Energía y Gas: www.creg. goc.co/index.php/es/sectores/energia/zni-energia

Ecomochilas.com. (2014). Ruta Macuira, The Desert with Cloud Forest at Sea Level. Obtenido de http:// www.ecomochilas.com/ruta-macuira/

Esteve Gómez, N. (2011). Energización de las zonas no interconectadas a partir de las energías renovables 
solar y eólica. Bogotá D.C.: Pontificia Universidad Javeriana.

Fernández Robles, J. (2017). Energías renovables en Colombia. Bogotá: Embajada de España en Colombia.

Figueroa, A., \& Mejía, E. (Julio-Diciembre de 2014). Sistemas híbridos: alternativa de energización en zonas no interconectadas. Revista de Estudios Interdisciplinarios en Ciencias Sociales, Tecnología e Innovación, 1(1), 44 - 64.

IDEAM. (s.f.). Atlas del Viento de Colombia. Recuperado el 19 de Enero de 2018, de http://atlas.ideam.gov. co/visorAtlasVientos.html

IPSE. (2018). Sistema de Información Geográfico del IPSE. Obtenido de http://190.216.196.84/ps_cnm/

Jiménez Pérez, F. (2015). Internado Indígena de Nazareth Ruta de la Pedagogia Amigoniana. Obtenido de http://slideplayer.es/slide/6161234/

Ministerio de Cultura. República de Colombia. (30 de julio de 2010). Caracterización del pueblo Wayuú. Wayuú, gente de arena, sol y viento. Obtenido de https://goo.gl/xgqCSs

NASA. (s.f.). NASA Surface meteorology and Solar Energy: RETScreen Data. Recuperado el 26 de enero de 2018, de https://goo.gl/NtdDrS

NREL. (s.f.). NSRDB Data Viewer. Recuperado el 22 de enero de 2018, de https://goo.gl/VkN9ux

OCHA. (2014). Informe Final MIRA: Alta Guajira Uribia (La Guajira), Colombia. Desabastecimiento de alimentos en comunidades Wayúu de la Alta Guajira. Equipo Humanitario Colombia.

Ojeda, E., Candelo, J., \& Silva, J. (2017). Perspectivas de Comunidades Indígenas de La Guajira Frente al Desarrollo Sostenible y el Abastecimiento Energético. Revista Espacios, Vol. 38(11), 25-43.
Ramírez Álvarez, M. E. (10 de Febrero de 2015). Viaje a la alta Guajira colombiana. Recuperado el 11 de Enero de 2018, de Las Dos Orillas: https://goo.gl/ g5paGn

SIPG. (s.f.). Precios de ACPM - Principales ciudades. Recuperado el 01 de Febrero de 2018, de https://goo.gl/ fSTn1k

Unidad de Planeación Minero Energética - UPME. (2015). Integración de las energías renovables no convencionales en Colombia. (M. d. Energía, Ed.) Bogotá: Ministerio de Minas y Energía. Recuperado el 10 de Enero de 2018

Unidad de Planeación Minero Energética (UPME). (2017). Plan de Expansión de Referencia Transmisión. Bogotá: Ministerio de Minas y Energía.

UPME - IDEAM. (2005). Atlas de Radiación Solar en Colombia. Bogotá: Ministerio de Minas y Energía.

UPME - IDEAM. (2006). Atlas de Viento y Energía Eólica de Colombia. Bogotá: Ministerio de Minas y Energía. 


\section{SOBRE LOS AUTORES:}

Jesús Antonio Valencia Plaza: Ingeniero Electrónico de la UNIAJC. Correo electrónico: valenciajesus03@gmail.com

Lida Soraida Lucano Mafla: Ingeniera Electrónica de la UNIAJC. Correo electrónico: liidita.lucano975@hotmail.com

Francisco Giovanni Ortega Palacios: Ingeniero Electrónico de la UNIAJC. Correo electrónico: fragorte@hotmail.com
Erika Sarria Navarro: Ingeniera Electrónica y Especialista en Redes de Comunicación. Docente Tiempo Completo UNIAJC. Correo electrónico: esarrian@admon.uniajc.edu.co

Carlos Mario Yepes: Ingeniero Electrónico y Magister en Energías Renovables y Eficiencia Energética. Docente Hora Cátedra UNIAJC. Correo electrónico: cgiraldo@eneco-ic.com

Andrés Mauricio González Moreno: Ingeniero Electrónico y Magister en Ciencias de la Educación para el Desarrollo Sostenible. Docente Hora Cátedra UNIAJC.Correo electrónico: agonzalez@ eneco-ic.com 